\title{
La querelle entre Bretons et Languedociens à propos du commerce de la sardine pressée au XVIIIe siècle
}

\section{Dominique Robin}

\section{OpenEdition Journals}

\section{Édition électronique}

URL : http://journals.openedition.org/abpo/1113

DOI : $10.4000 /$ abpo. 1113

ISBN : 978-2-7535-1499-7

ISSN : 2108-6443

Éditeur

Presses universitaires de Rennes

Édition imprimée

Date de publication : 20 septembre 2005

Pagination : 19-36

ISBN : 978-2-7535-0201-7

ISSN : 0399-0826

\section{Référence électronique}

Dominique Robin, « La querelle entre Bretons et Languedociens à propos du commerce de la sardine pressée au XVIIIe siècle », Annales de Bretagne et des Pays de l'Ouest [En ligne], 112-3 | 2005, mis en ligne le 20 septembre 2007, consulté le 19 avril 2019. URL : http://journals.openedition.org/abpo/1113 ; DOI : 10.4000/abpo.1113 


\title{
La querelle entre Bretons et Languedociens à propos du commerce de la sardine pressée au XvIII ${ }^{\mathrm{e}}$ siècle
}

\author{
Dominique RoBin \\ Agrégé et docteur en histoire
}

On trouve aux Archives départementales d'Ille-et-Vilaine un dossier conservé par les services de la Commission Intermédiaire des États de Bretagne ${ }^{1}$. Ce dossier, constitué en 1767, fait état d'une longue polémique particulièrement vive, bien qu'uniquement épistolaire, opposant, au $\mathrm{XVIII}^{\mathrm{e}}$ siècle, Bretons et gens du Languedoc. L'objet du différend est le commerce de la sardine. Les Méditerranéens sont accusés par les négociants bretons en sardines, tous établis sur les ports de la côte atlantique, de leur faire une concurrence déloyale par des importations durables et massives de sardines de pêche étrangère.

En dehors d'un texte qui date du $1^{\mathrm{er}}$ septembre 1767 et qui est à l'origine de tout ce dossier, on trouve, dans la même liasse, deux autres documents datant de 1749 et de 1754 qui ont pour but d'étayer et de justifier les plaintes de 1767. Le document de 1749 est une copie d'une requête de l'intendant du Languedoc, Le Nain, adressée au Contrôleur Général des Finances. Cet intendant sollicite, au nom des négociants en sardines des côtes du Languedoc, la permission d'importer des sardines de pêche espagnole ou portugaise. Cette demande a suscité immédiatement une réponse vive de la part des négociants bretons : ceux-ci répondent point par point aux arguments circonstanciés de l'intendant Le Nain. Le document de 1754 est, quant à lui, une " réponse des Bretons " s'adressant cette fois-ci surtout aux Basques, autres solliciteurs de pêche étrangère, mais il y est fait souvent allusion aux gens du Languedoc.

Cette polémique entre Bretons et Languedociens peut surprendre dans une société d'Ancien Régime. En effet, tout sépare alors ces deux provinces : le climat, le mode de vie et surtout les distances, considérables et difficilement surmontables en raison des carences ou des lenteurs des

1. Arch. dép. d'Ille-et-Vilaine, C 3928. Créée en 1734, la Commission Intermédiaire représente un rouage permanent et essentiel des États de Bretagne. 
moyens de transport. Cette querelle est d'autant plus surprenante qu'il s'agit d'un sujet concernant un produit hautement périssable, la sardine, et donc condamné, semble-t-il, à un commerce de proximité. Alors, comment comprendre cette confrontation entre deux régions qui sont appelées à s'ignorer?

\section{Les caprices de la nature perturbent des équilibres séculaires}

Deux faits vont perturber l'équilibre traditionnel en matière de commercialisation de la sardine. Il y a d'abord la question des variations de la sardine de Méditerranée. Le début du XVIII ${ }^{\mathrm{e}}$ siècle s'avère catastrophique pour les pêcheurs de sardine sur les côtes méditerranéennes de France. Depuis plusieurs décennies, ce poisson y fait cruellement défaut. C'est une véritable catastrophe dans le Midi, d'abord pour les pêcheurs et négociants mais aussi pour la population la plus pauvre qui est une grande consommatrice de ce poisson bon marché.

Le mémoire adressé en 1749 au Contrôleur Général par M. Le Nain, intendant de Languedoc, donne la mesure de ce qui peut être une catastrophe dans une société d'Ancien Régime où la pénurie alimentaire est chose courante, surtout pour les plus faibles :

"La pêche de ce Poisson était autrefois très abondante à Cette en Languedoc ${ }^{2}$; on y en salait au-delà de ce qui était nécessaire pour la consommation de cette Province : mais il y a environ vingt-cinq ans qu'elle a manqué. Les sardines ne s'arrêtent plus sur cette Côte, comme elles faisaient autrefois depuis le commencement du Printems jusqu'à la fin de l'Automne. On en voit bien paraître toutes les années : elles passent de suite en Catalogne, cependant elles sont absolument nécessaires pour la nourriture des Pauvres, surtout pour les gens de la Campagne ${ }^{3}$."

Le Nain insiste sur les répercussions au niveau social de la disparition de la sardine sur les côtes languedociennes :

«La perte du Salage de Cette s'y est extrêmement faite sentir, les Pauvres en ont beaucoup souffert, surtout depuis la guerre ${ }^{4}$, où la Moruë sèche qui pouvait y suppléer en partie a totalement manqué. Il restait cependant une ressource : la sardine qui ne fait plus que passer sur les Côtes du Languedoc, s'arrête en Catalogne, où on a grand soin de la faire saler, ou pour le compte des Marchands Languedociens ou pour celui des Catalans. Il s'en consomme une partie sur les lieux, et le reste arrive dans les Ports de Languedoc, d'où il se répend dans toute la Province, et fournit à la nourriture des Païsans. "

Comment expliquer cette soudaine et durable désaffection de la sardine sur les côtes du Languedoc? L'explication viendrait des caprices du Rhône :

" Il y a environ quarante ans que le cours du Rhône, devenu plus rapide qu'il l'était avant, repoussa et fit dériver sur les Côtes de Catalogne, la

2. Actuellement Sète.

3. Arch. dép. d'Ille-et-Vilaine, C 3928.

4. Nous sommes en 1749, il s'agit donc de la guerre de Succession d'Autriche. 
Sardine qui abondait sur celles du Languedoc et dont il se faisait à Cette un salage considérable, tant en presse qu'en saumure, mais qui depuis cette époque se trouve réduit à peu de chose."

L'explication vaut ce qu'elle vaut, toujours est-il que la désaffection de la sardine est bien une réalité et qu'elle a des conséquences énormes pour l'économie locale.

Le second fait, qui va perturber l'équilibre traditionnel du commerce de la sardine résulte du premier. Beaucoup de négociants du Languedoc, las d'attendre un poisson qui ne vient plus, se résignent finalement à émigrer en Bretagne ou à l'étranger pour conserver la mainmise sur le commerce et l'industrie de la sardine pressée, celle qui était habituellement consommée dans le Midi :

"Une partie de ceux qui s'occupaient à ce salage vinrent, tant de Marseille que de Cette et Montpellier, s'établir en Bretagne pour les mêmes opérations $^{5}$. "

Ce déplacement vers la Bretagne a dû être quelque peu provoquée par les offres de la famille très puissante des Fouquet, alors propriétaire de Belle-Île. Le fait est qu'il y a bien eu une première vague d'immigration de Méridionaux en direction de cette île dès le XVII ${ }^{\mathrm{e}}$ siècle. C'est ainsi que s'est faite, à Belle-Ille et dans le quartier maritime de Port-Louis, la première installation des presses à sardines en Bretagne.

Vers 1730, arrive en Bretagne, toujours dans la même zone, une autre vague de Méridionaux. La pénurie de sardines sur les côtes françaises de la méditerranée ne cesse, en effet, de s'accroître. Le Nain, l'intendant du Languedoc, s'en fait le témoin en 1749 :

" Cette espèce de nécessité et la grande consommation qui se fait de cette denrée, engagèrent les Marchands Saleurs de Cette et de Montpellier d'en aller faire saler à Bel'Isle; lorsqu'on fut bien convaincu qu'il n'y avait plus à compter sur celle de Cette ${ }^{6}$."

Forts d'un savoir-faire ignoré jusque-là en Bretagne, ces négociants du Midi ont pour effet de relancer l'activité sardinière en Bretagne en lui donnant une vocation commerciale de grande ampleur, vocation qu'elle n'avait jamais eue auparavant. En effet, il apparaît bien que l'art du salage de sardines de longue garde - il est question ici de la conservation de sardines pressées - était inconnu des Bretons qui se contentaient jusqu'ici de commercialiser des sardines en vert ${ }^{7}$. Ainsi, grâce à l'apport de ces négociants méridionaux, la Bretagne est devenue, et pour longtemps, la grande région de production de sardines pressées en France. L'arrivée inopinée de ces négociants a été, sans aucun doute, le coup de fouet nécessaire à l'expansion de la pêche de la sardine bretonne : désormais, s'ajoute à la sardine en vert, jus-

5. Arch. dép. d'Ille-et-Vilaine, C 3928.

6. Arch. dép. d'Ille-et-Vilaine, C 3928, 1749.

7. Sardines fraîches, recouvertes d'une simple couche de sel pour la conservation pendant le voyage. 
qu'ici fleuron de la pêche atlantique bretonne, la sardine pressée, aux perspectives commerciales beaucoup plus larges et beaucoup plus assurées. Ces sardines peuvent en effet se conserver sur une période de sept à huit mois.

Cette activité florissante en Bretagne reçoit, dès lors, une aide précieuse de l'État pour l'accroissement de la pêche de la sardine. L'aide de l'État est décisive en particulier du fait de l'arrêt du Conseil du 24 août 1748 qui donne aux Bretons, dans tout le royaume, un quasi monopole de la commercialisation de la sardine de pêche nationale. Cet arrêt a engagé les plus hautes autorités de l'État. Phélypeaux, Comte de Maurepas, en est le signataire en tant que Secrétaire d'État de la Marine mais à ses côtés ce sont les plus hauts dignitaires de l'État qui se sont engagés aussi dans cette action :

"Sa Majesté étant informée que, nonobstant ces défenses, il a été introduit dans quelques Provinces du Royaume, sous différens prétextes, des Sardines de pêches étrangères, à quoi voulant pourvoir : vû l'avis des Députés du Commerce, Ouï le Rapport du sieur de Machault Conseiller ordinaire au Conseil Royal, contrôleur général des Finances, le Roy étant en son Conseil, a ordonné et ordonne que lesdits arrêts de son conseil seront exécutés selon leur forme et teneur; en conséquence, fait Sa Majesté itératives et très expresses inhibitions et défenses à tous Capitaines de Navires, Maîtres de Barques, Négocians et autres, d'introduire et faire entrer dans le Royaume, des Sardines de pêches étrangères, de quelques pays qu'elles puissent venir, à peine de confiscation desdites Sardines et en outre de trois mille livres d'amende contre chacun des contrevenants et pour chaque contravention ${ }^{8}$."

L'État y trouve son intérêt : il peut voir dans cet encouragement à la pêche bretonne un excellent moyen de recruter à bon compte de nouveaux marins pour la Royale en cas de nouvelles guerres navales contre l'ennemi d'alors, l'Angleterre. La position géographique de la Bretagne qui fait de celle-ci une forteresse avancée face à l'ennemi héréditaire, ne peut que plaider en faveur de la montée en puissance d'une Bretagne maritime, alors insuffisamment représentée sur la côte sud.

Mais la monarchie a sans doute mal mesuré les conséquences de cette mesure de protection des pêches nationales. Elle est à l'origine du conflit entre les deux provinces de Bretagne et du Languedoc. En effet, en faisant de la Bretagne le seul pourvoyeur en sardines du Languedoc, la monarchie risque de priver les provinces méridionales d'autres sources d'approvisionnement beaucoup plus proches, venant en particulier d'Espagne, du Portugal, du Comté de Nice. Que faire si la Bretagne, désormais fournisseur exclusif du Languedoc en sardines pressées, est incapable de satisfaire une clientèle constamment dans le besoin?

Le conflit est donc alimenté par une situation nouvelle dans le royaume : la présence d'une région productrice mais faible consommatrice de sardines, la Bretagne, et celle d'une autre région, momentanément faible productrice et toujours grande consommatrice, le Languedoc. Cette dichoto-

8. Arch. dép. du Morbihan, B 2137, 24 août 1748. 
mie au sein du royaume est à l'origine de querelles permanentes entre les deux provinces, querelles dont un des sommets se situe justement en 1767.

\section{Les Bretons sont les producteurs et les gens du Languedoc sont les consommateurs}

Pour bien comprendre ces querelles répétées, et les différentes stratégies employées par les deux provinces, il faut savoir que nous sommes dans un monde où, la précarité alimentaire s'affirme partout et toujours; les producteurs sont pratiquement incapables de répondre aux exigences d'une population toujours à la recherche de la satisfaction de ses besoins les plus élémentaires. Pour cette raison, les producteurs bretons de sardines pressées, livrés aux aléas d'une conjoncture qu'ils sont incapables de maîtriser, subissent constamment la pression et les exigences des consommateurs languedociens insatisfaits. Fernand Braudel parle de « l'offre qui se présente, à la fin du XVIII ${ }^{\mathrm{e}}$ siècle, devant l'ogresse qu'est la consommation ${ }^{9}$ "; l'offre est défaillante, " chétive et discrète " pour reprendre les mots de Braudel. Ainsi, la Bretagne, pourtant a priori détentrice des sources de richesse, loin de dicter sa loi est en fait constamment sur la défensive et doit en permanence faire face aux menaces réitérées du Languedoc.

Depuis l'arrivée des méridionaux en Bretagne, la pêche bretonne de la sardine entre dans un cadre encore élargi de ses activités marchandes. Désormais l'ambition n'est plus, grâce aux chasse-marée, grands rouliers des mers dans ce domaine, d'approvisionner en sardines en vert et d'une manière saisonnière, une toute petite tranche de population proche des littoraux de l'Atlantique, mais d'assurer, pendant plusieurs mois, la satisfaction de la clientèle du Languedoc, grosse consommatrice de sardines pressées. Pour les Bretons, les enjeux ont changé de dimensions, il faudra se montrer à la hauteur de la tâche. C'est là que commencent les problèmes.

Il faut d'abord faire front à une pêche qui est constamment aléatoire. La pêche de la sardine nous plonge dans le monde du précaire : les caprices de fortune de pêche en sont le dénominateur commun. Les responsables sont d'abord la sardine elle-même : celle-ci est, par excellence, un poisson capricieux : rien ne ressemble moins à une année de pêche qu'une autre année de pêche. Les hommes, de leur côté, ont mis aussi un certain acharnement à accentuer la précarité du métier de pêcheur et à perturber son rythme de travail : les guerres maritimes entreprises par l'État, par exemple, ont profondément affecté les rendements des pêcheurs tout au long du XVIII ${ }^{\mathrm{e}}$ siècle.

Le tableau qui suit, portant sur les pêches bretonnes de 1740 à 1789 , montre les difficultés à exploiter la ressource en sardines d'une manière régulière. Sur vingt-huit années de pêche répertoriées, on en compte treize qualifiées de correctes, douze de pénurie et trois de médiocres. Les années correctes sont donc aussi nombreuses que les années de pénurie mais cela

9. BRAUDEL, Fernand, Civilisation matérielle, économie et capitalisme, XVe-XVII siècle, t. II, p. 200. 
ne veut pas dire grand-chose, d'autant plus que des années ont été considérées comme correctes en temps de guerre, seulement parce que le poisson était abondant et beau. Il faudrait alors tenir aussi compte du nombre de marins disponibles ces années-là. Ce qui compte, c'est de voir dans quelles circonstances arrivent les bonnes et mauvaises années. On peut voir que dans les années 1740 et 1750, le bilan général est mitigé : il y a autant de bonnes que de mauvaises années de pêche mais les années mauvaises sont particulièrement désastreuses, c'est le cas pour les années 1741 et 1755 . Ensuite, il y a une bonne période, dans la décennie qui suit la guerre de Sept Ans. En revanche les années 1770 et le début des années 1780 sont particulièrement calamiteuses. Elles arrivent au plus mauvais moment, c'est-à-dire au moment où les récoltes de grains sont, elles aussi, mauvaises. Cette conjonction des malheurs ne peut avoir que des incidences désastreuses sur le comportement des populations les plus pauvres, celles qui se nourrissent uniquement, faute de mieux, de sardines et de céréales.

Tableau 1 - Bonnes et mauvaises années de pêche

\begin{tabular}{|c|c|c|}
\hline Bonnes Années & Mauvaises Années & Années Médiocres \\
\hline & 1741 & \multirow{13}{*}{1760} \\
\hline 1748 & & \\
\hline 1749 & & \\
\hline 1750 & & \\
\hline & $\begin{array}{l}1751 \\
1752\end{array}$ & \\
\hline 1754 & & \\
\hline & 1755 & \\
\hline $\begin{array}{l}1758 \\
1759\end{array}$ & & \\
\hline \multirow{10}{*}{$\begin{array}{l}1764 \\
1765 \\
1766 \\
1767 \\
1769\end{array}$} & & \\
\hline & & \\
\hline & & \\
\hline & & \\
\hline & & \\
\hline & & \multirow[t]{4}{*}{1770} \\
\hline & 1771 & \\
\hline & 1772 & \\
\hline & $\begin{array}{l}1773 \\
1777\end{array}$ & \\
\hline & & \multirow[t]{5}{*}{1778} \\
\hline \multirow{2}{*}{1779} & 1700 & \\
\hline & 1781 & \\
\hline \multirow[t]{2}{*}{1783} & & \\
\hline & $\begin{array}{l}1784 \\
1785\end{array}$ & \\
\hline
\end{tabular}

Dans ces conditions, il n'est pas étonnant de voir que les Bretons doivent sans cesse répondre aux critiques des consommateurs du Languedoc. Ainsi, l'intendant de Bretagne, de Viarmes, s'adressant au Contrôleur général des Finances Machault d'Arnouville, cherche à minimiser le problème 
en affirmant, en mars 1749, que cette question de l'insuffisance de l'offre n'est qu'un faux problème.

Pour l'intendant de Viarmes, la Bretagne " produit depuis quelques années beaucoup plus qu'il n'en faut pour la consommation du Royaume $^{10}$ ". L'intendant de Bretagne, qui a bien étudié le sujet, continue son propos en s'appuyant sur une documentation fournie :

" Il s'y fait des sardines anchoitées qui se mettent en petits barils et que du seul port du Port-Louis il est sorti en 1748, pour le procurer à ceux du Languedoc, près de 17000 petits barils, que Belle-lle, Concarneau, Audierne, Douarnenez, Camaret et les autres ports en auront au moins fournis chacun autant, quantité plus que suffisante pour la consommation de ces provinces, et présentement que la tranquilité est rendue à l'Europe et que nos côtes sont libres ce commerce ne peut que doubler. Le progrès que cet arrêt a occasionné dans cette partie du Commerce est sensible. On estime qu'il est sorti de Bretagne cette année plus de 80000 petits barils de sardines anchoitées et plus de 25000 barriques pressées et les négocians comptent doubler leurs envoys ou du moins les augmenter du tiers ${ }^{11}$."

Son propos n'est pas dénué de tout fondement. En effet, à cette époque, la pêche de la sardine, aiguillonnée par le succès des presses bretonnes, voit ses effectifs augmenter d'une manière manifeste, tant dans l'amirauté de Vannes que dans celle de Quimper. On peut en juger à partir des documents qui suivent. Sur la première figure, le nombre de chaloupes augmente d'une manière considérable entre 1728 et 1754 puisqu'il passe de 700 à 1137 , soit une augmentation de la flottille des deux tiers en seulement vingt-cinq ans (Figure 1). L'île de Groix est le lieu où le succès est le plus foudroyant, de 1728 à 1749, en vingt ans seulement, la flottille de pêche à la sardine a plus que doublé (Figure 2). Cette progression est vraiment durable, ainsi en 1767, Besson, observe les progrès continus de cette pêche :

" Il y a vingt ans que l'on comptait à peine moitié du nombre de négotiants de presses ou de chaloupes de peche que l'on voit aujourdhuy : on peut en général y assurer que si par quelque moyen on ajoutait quatre matelots à une paroisse on verrait aussi tot une chaloupe de plus. Ce commerce ne sera jamais borné que par la dizette d'hommes ou par les difficultés qu'ils trouveront d'y gagner leurs vies comme par le passé ${ }^{12}$."

La situation de Concarneau (Figure 3), dans l'amirauté de Quimper, où le succès est plus long à venir, illustre parfaitement ces derniers propos quand on considère les progrès foudroyants de la fin du siècle : ils montrent les énormes potentialités offertes par l'océan sur les côtes bretonnes en matière de ressources en sardines ${ }^{13}$.

10. Arch. dép. d'Ille-et-Vilaine, C 1595, 20 mars 1749.

11. Arch. dép. d'Ille-et-Vilaine, C 1595.

12. Arch. dép. d'llle-et-Vilaine, C 3928, 14 août 1767.

13. Pour une étude plus détaillée voir RoBin, Dominique, La Pêche en Bretagne sous l'Ancien Régime : l'exploitation de la sardine sur la côte atlantique, Presses Universitaires de Rennes, 2000, 387 p. 
Figure 1 - La pêche à la sardine en Bretagne, nombre de chaloupes au XVIII siècle

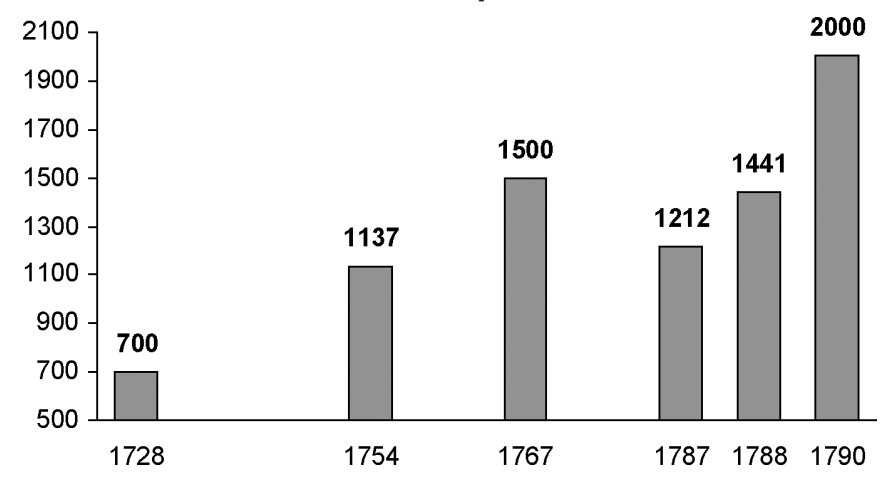

Figure 2 - La pêche à la sardine à Groix, nombre de chaloupes au XVIII ${ }^{e}$ siècle

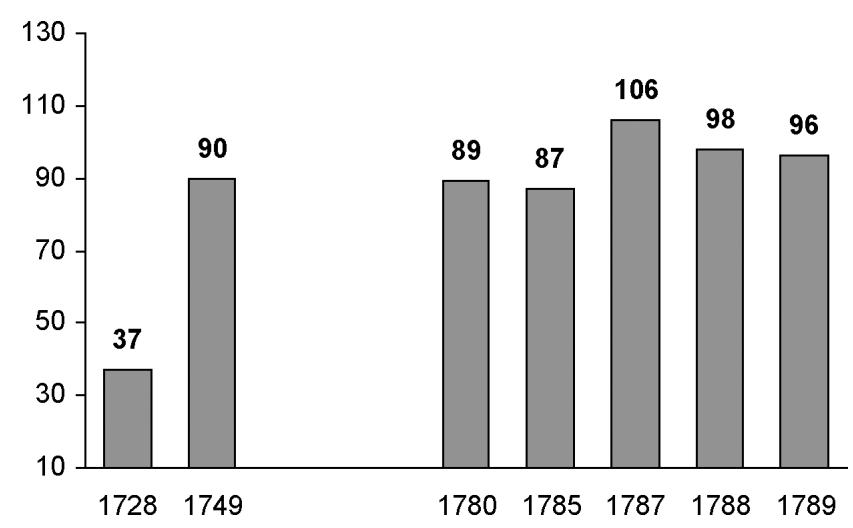

Figure 3-La pêche à la sardine à Concarneau, nombre de chaloupes au XVIII siècle

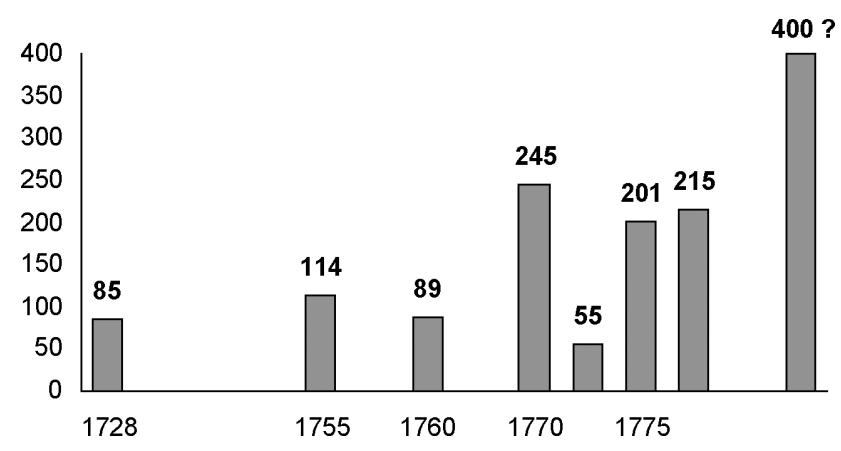


Mais tout cela ne suffit pas à apaiser les craintes des méridionaux qui émettent aussi certaines critiques au sujet de la qualité du produit. N'ayant plus de sardines chez eux, ils préfèrent se rabattre sur les sardines de Catalogne dont, pour des raisons techniques, la qualité est plus assurée. L'intendant Le Nain, dans son mémoire de 1749 ne manque pas de mettre en évidence les carences de la Bretagne :

"Le salage de Catalogne se fait de la même manière que celui de Cette, c'est-à-dire, en petits barils ou demi barils, ce qui est très commode pour la vente, et le Poisson d'ailleurs s'en conserve mieux. Les sardines qu'on sale à Bel'Isle sont d'abord mises dans des grosses bariques ou tonneaux, pour la facilité du transport; après quoi il faut les en tirer et les remanier pour les mettre en petits barils; sans cela il n'y aurait pas moïen de les vendre en détail. Cette seconde opération déprécie beaucoup ce salage; le poisson ne sçaurait être ni si beau ni si bon, ni si entier que lorsqu'il a été d'abord salé en petits barils. Ceux-ci sont appellés barils de Mère et les autres barils refaits ${ }^{14}$."

La réponse des Bretons, toujours sur la défensive, ne tarde pas :

"On n'est pas bien informé quand on objecte que les Sardines qu'on sale à Belle-Isle (on aurait dû dire en Bretagne) sont d'abord mises dans des grosses barriques, pour être ensuite transvasées dans le Languedoc en petits barrils; le fait est vrai pour une partie du salage qui se fait en Bretagne pour la commodité du pauvre du Languedoc : mais il n'est pas moins vrai pour celle du riche, que d'Olonne, Belle-Isle, Etel, Port-Louis et Concarneau seulement, il s'en est fait au moins, année commune, cent mille barrils de mer pour la provision du Languedoc. Les Saleurs de cette Province établis en Bretagne et les autres, sont en état d'en fournir le double, si la consommation s'en pouvait faire ${ }^{15}$."

Les Bretons n'acceptent pas cette concurrence étrangère, venant d'Espagne ou du Portugal, voire du comté de Nice :

" La Catalogne ou le Comté de Nice, où on ne sale que peu de Sardines, une fois exceptée des défenses, deviendra sans contredit l'Entrepôt de toutes les Sardines, non seulement qui se pêcheront aux Côtes d'Oran et de Portugal, mais de celle de l'Angleterre même, comme il est arrivé si souvent. Dans ces Entrepôts il se formera un déluge de sardine qui inondera le Languedoc et autres Provinces, au grand préjudice de la pêche de Bretagne, qui se trouvera par là ruinée et anéantie sans ressource, puisqu'on lui aura ôté le débouchement de ses Sardines, qu'elle ne trouve, pour ainsi dire, que dans ces seules Provinces ${ }^{16}$."

En 1754, le mémoire intitulé Réponse des Bretons est une réplique à un mémoire du pays de Labourd de 1753, autre province consommatrice de sardines tentée par l'aventure des importations de sardines espagnoles. Ce mémoire de 1754 veut mettre fin à cette accusation d'une Bretagne incapable d'assurer l'approvisionnement en sardines des populations consommatrices du Midi :

14. Arch. dép. d'Ille-et-Vilaine, C 3928, 1749.

15. Arch. dép. d'Ille-et-Vilaine, C 3928.

16. Arch. dép. d'Ille-et-Vilaine, C 3928. 
"Un Particulier de Montpellier, qui l'année dernière tira de Saint-Jeande-Luz pour 20000 liv. de Sardine, a laquelle la faveur du bon marché luy fit donner la préférence sur celle de Bretagne, y perdit au moins les deux tiers de son capital. L'abondance des Sardines d'Espagne et de Bretagne devint si grande l'hyver dernier en Provence et en Languedoc, qu'il en est resté plusieurs parties des unes et des autres qui sont actuellement invendues à Cette et en risque d'être jettées a la mer, seule, mais triste ressource d'une denrée sujette à la corruption et sur laquelle la Police est souvent obligée d'exercer son ministère, surtout lorsque l'abondance est portée au delà de la consommation. "Marchand de Sardines, marchand d'Oranges", c'est un ancien proverbe, qui tire son origine d'une Comparaison bien juste, et qui ne s'est que trop vérifiée depuis que les Espagnols ont trouvé chez les Basques un débit abondant et aisé de leur Sardine, contre les dispositions de l'arrêt du Conseil qu'on a déjà cité, et qui n'estoit que la Confirmation de celui du 18 novembre $1720^{17}$."

Ces querelles cachent d'autres objectifs, moins avouables, que ceux de la seule lutte contre la précarité. Les négociants du Midi exercent une pression de tous les instants sur leurs fournisseurs, les négociants bretons, pour garder le contrôle du marché de la sardine : ils ont accepté de perdre la ressource en sardines, il n'est pas question d'en perdre en plus le marché.

Cette pression se fait en deux temps. Les pêcheurs bretons sont rarement en contact direct avec les Languedociens. Les Bretons, préfèrent laisser cette tâche aux négociants de Bordeaux qui leur servent d'intermédiaires entre la Bretagne et la Méditerranée. Pourquoi Bordeaux? Parce que de tout temps il y a eu des relations entre négociants des ports bretons et bordelais, en particulier dans l'amirauté de Vannes. Mais cet intérêt grandissant de certains négociants de Bordeaux pour la pêche de la sardine en Bretagne s'explique surtout par la position stratégique qu'offre leur ville. Le port de Bordeaux sert de transit entre les lieux de production, la côte sud de la Bretagne, et les véritables lieux de consommation de sardines, le Midi méditerranéen. La ville se trouve, grâce à la Garonne et au canal du Midi, alors nouvellement ouvert, à la tête d'un vaste hinterland qui prolonge l'influence du bassin de la Garonne bien au delà de ses limites naturelles. Le canal du Midi ouvre à Bordeaux les portes du monde méditerranéen et il joue tout particulièrement un rôle essentiel pour l'acheminement des sardines pressées de Bretagne vers les régions les plus méridionales qui sont traditionnellement de grosses consommatrices de sardines : les deux provinces du Languedoc et de la Provence consomment à elles seules autant de sardines pressées venant de Bretagne que tout le reste de la France.

De ce fait, les Languedociens font pression sur les négociants bordelais, et non sur les Bretons, pour que l'acheminement des sardines pressées soit conforme à leurs souhaits. Ces derniers répercutent aux Bretons les ordres du Languedoc. Pour illustrer tout cela, on peut prendre les propos tenus, en janvier 1758, par Groc, négociant de Bordeaux, à l'intention de son cor-

17. Arch. nat. Marine, C 5 36, 1754. 
respondant de Concarneau, Allix. Le négociant de Bordeaux lui fait part de la pression des ordres venant du Languedoc :

" Je me règle sur les ordres que j'ay du Languedoc pour de la sardine et prévoyant que notre presse ne sera pas assés suffisante pour les effectuer, je viens vous dire de travailler au reçu de ma lettre et sans mener grand bruit à m'en faire l'achat de 200 demy bariques au cours le plus bas qu'il se pourra en vous attachant aux meilleures presses et à la plus belle jauge ${ }^{18}$. "

Par la suite, en juin 1758, au moment où il conseille à Allix de veiller à la bonne opération du pressage des sardines, Groc nous donne une description plus précise de l'aire géographique avec laquelle il est en contact commercial. Il s'agit de tout le Languedoc dans l'acception territoriale la plus vaste de la Province :

"Comme ce poisson est quelque fois corrompu avant qu'il ne soit rendu aux scelliers par les divers chaleurs qu'il y fait et que par cette raison elle est plus en état de s'éventrer qu'en septembre et octobre il faut avoir attention qu'on ne le fasse pas autant presser qu'on le fait au poisson pêche d'hiver car autrement se serait vouloir m'exposer à recevoir des reproches de ceux à qui je les envoye dans le haut et Bas Languedoc ${ }^{19}$. "

Quelques mois plus tard, en décembre 1758, Groc fait part à Allix de ses inquiétudes à propos du prix exorbitant des sardines pressées en Bretagne. Les gens du Languedoc, une fois de plus, sont là pour rappeler leurs exigences. C'est sous la pression des consommateurs Languedociens que Groc doit surveiller les prix de la sardine qui est un produit de consommation pour gens du peuple; il faut donc être attentif au fait que les prix ne soient pas excessifs :

"Vous me dittes que la sardine vaut chés les pêcheurs de 74 à 75 livres. Combien vaudra-t-elle donc chés les fabriquans? En vérité il y a de la folie de la part de ceux qui la font valoir autant [...] C'est vouloir perdre son bien de gayeté de cœur, puisque dans le Languedoc et à Toulouse on n'en veut pas seulement à 85 livres $^{20}$."

Le 22 janvier 1759, la période du carême approche, c'est une période où la consommation de sardine atteint un pic par rapport au reste de l'année. À nouveau, on retrouve dans les propos de Groc le souci qu'il a de ne pas décevoir ses clients Languedociens :

" Je suis inquiet de voir que vous n'ayés pu encore me charger le restant de mes sardines, tachés donc d'y parvenir au plutot si vous ne l'avés déjà fait. Sans cela je me vois exposé à perdre gros si je ne les reçois même que 15 jours avant le carême, attendu que n'ayant pas assés de temps pour pouvoir les envoyer à ceux du Languedoc qui m'en demandent et tous nos marchans peu ou prou en ayant fait venir, je seray obligé d'attendre qu'ils ayent vendu la leur à leur détail pour leur vendre ensuitte la mienne ${ }^{21}$."

18. Arch. dép. du Finistère, Brest, B 4619, 24 juillet 1758.

19. Arch. dép. du Finistère, Brest, B 4619, 10 juin 1758.

20. Arch. dép. du Finistère, Brest, B 4619, 11 décembre 1758.

21. Arch. dép. du Finistère, Brest, B 4619, 22 janvier 1759. 
Les Bretons, bien que pêcheurs et uniques producteurs de sardines pressées du royaume, se trouvent en fait au bout de la chaîne. Ils sont bien sous la domination de la demande et en sont réduits, bien souvent, à trouver des excuses piteuses lorsque, par exemple, le prix de la sardine est jugé trop élevé par les gens du Languedoc. Il est alors de bon ton d'incriminer les bureaux de la Ferme qui cristallisent autour d'eux un impopularité générale partout dans le royaume :

"Si les pauvres du Languedoc souffrent, ce ne peut être que de la perception du droit intolérable de 10 sols 4 deniers par barril de sardine en saumure, et de 6 livres 6 sols par barrique de sardine pressée, qui se fait à l'entrée de la Province dans les deux Bureaux que le Fermier a établi impunément, l'un au Village d'Ondes sur la Garonne, et l'autre au lieu de Labarthes sur la Rivière du Tarn. L'objet qui tend au soulagement du pauvre est très louable; mais est-ce le remplir que de passer sous silence le poids onéreux d'un nouveau droit qui retombe sur lui et dont le Fermier a respecté si long-temps la franchise ${ }^{22}$ ?"

En dépit de la législation royale en faveur de la protection des pêches nationales, les négociants du Languedoc, soutenus par leur intendant, veulent faire fléchir les autorités de Paris en faveur de règles plus souples. La situation de pénurie que subissent les couches les plus populaires du Languedoc devrait permettre à la concurrence de régler les carences de l'approvisionnement breton en sardines. Faire jouer la concurrence c'est donc accepter de faire venir des sardines de pêche étrangère. Il se trouve que la sardine méditerranéenne continue à ranger les côtes de Catalogne et d'Espagne et que le long des côtes atlantiques du Portugal la ressource en sardines ne manque pas. Le Nain ne manque pas de faire valoir au Contrôleur Général des finances ces opportunités pour la région :

\begin{abstract}
"Mais comme elle manque aussi quelquefois en Bretagne, et que dans les années où elle est le plus abondante, il n'est pas possible qu'elle le soit assez pour fournir à la consommation de tout le Roïaume; on s'est tourné d'un autre côté pour y suppléer; et deux ou trois de nos Négocians se sont avisés d'essaïer d'en aller faire un salage en Portugal [...] il est bien certain qu'on doit favoriser les établissemens du Roïaume préférablement aux Etrangers : mais le Roïaume est bien étendu; et il faut prendre garde, en voulant faire du bien à une Province de ne pas nuire à d'autres; on est bien persuadé que ce n'est pas-là l'intention du Roi ni des Ministres : mais comme il n'est pas possible de voir tous les objets du même point de vuë, et que dans cette affaire on s'est uniquement attaché à faire valoir les intérêts de la Bretagne; il est arrivé que sans songer au Languedoc, on lui a porté un préjudice considérable, cette Province étant une de celles où il se fait une plus grande consommation de Sardine salée ${ }^{23}$."
\end{abstract}

Autrement dit, les gens du Languedoc demandent au moins la modification de l'arrêt du Conseil du 24 août 1748 qui interdit de consommer la sardine de pêche étrangère. Pour reprendre l'expression de Le Nain, la prio-

22. Arch. dép. d'Ille-et-Vilaine, C 3928.

23. Arch. dép. d'Ille-et-Vilaine, C 3928. 
rité " c'est de laisser les choses sur l'ancien pied "; il propose qu'on en revienne à un régime de liberté, celui qui précédait l'Arrêt du Conseil commandé par les Bretons sur la protection des pêches nationales.

\section{Les Bretons veulent mettre un terme à cette querelle}

La monarchie se trouve prise entre deux priorités contradictoires. D'un côté sa mission est de protéger les populations les plus pauvres du Languedoc, ne serait-ce que pour maintenir l'ordre social. Mais pour cela il faut accepter, au mépris de ses propres engagements faits au plus haut niveau, l'importation de sardines étrangères venant d'Espagne, du Portugal ou du Comté de Nice, sardines réclamées à cor et à cri par les autorités provinciales et les négociants du Languedoc. Quelle crédibilité peut alors avoir l'État, si ce dernier est incapable de faire respecter des Arrêts dont il est lui-même à l'origine? D'un autre côté, cette initiative met les autorités de la monarchie dans une position délicate en Bretagne car le risque est grand de voir alors dépérir, face à la concurrence étrangère, une industrie nouvelle et florissante qui s'appuie en bonne partie sur la protection des pêches nationales. Cela priverait en outre la flotte militaire d'un recrutement supplémentaire nécessaire pour le maintien de sa puissance.

Les Bretons, soutenus par la colère unanime de tous les négociants en sardines et par les autorités provinciales, en particulier par les États de Bretagne, veulent en finir au plus vite avec ces atermoiements. Nous en arrivons à la plainte du $1^{\mathrm{er}}$ septembre 1767. Les négociants du Port-Louis et de l'île de Groix sont à l'origine de cette plainte mais la plainte déborde largement cette petite aire géographique. Le mécontentement est général dans tous les ports sardiniers bretons de la côte atlantique :

" Nous négocians et Fabriquans susdits, tant pour nous que pour les Pêcheurs des Côtes circonvoisines, donnons pouvoir et procuration auxdits Sieurs Négocians et Fabriquans de Sardines à Concarneau de, pour nous et en notre nom, signer la Requête à présenter à Sa Majesté aux fins d'obtenir de sa Protection Royale en faveur des Commerçans de sa Province de Bretagne, des moyens d'empêcher sous aucuns prétextes l'entrée de toutes espèce de Sardine provenant de Pêche étrangère, dont l'introduction entraînerait infailliblement la ruine des Fabriquans Français et celle de plusieurs milliers de Pêcheurs qui ne vivent qu'à l'appui de ce commerce ${ }^{24}$."

En fin de requête on trouve les signatures de négociants en sardines d'au moins dix ports de pêche : le Port-Louis, l'île de Groix, Étel, Quiberon, Pont-L'Abbé, l'île Tudy, Penmarch, Le Guilvinec, Audierne et enfin Concarneau. Les deux ports les plus importants sont alors Concarneau et le Port-Louis. Manquent seulement Douarnenez Belle-lle et Le Croisic. C'est dire l'ampleur du mécontentement. Parmi les signataires bretons de 1767 on trouve aussi les plaintes de négociants d'origine méridionale dont les intérêts sont maintenant bien implantés en Bretagne, en particulier dans

24. Arch. dép. d'Ille-et-Vilaine, C 3928. 
le quartier maritime du Port-Louis et dans celui de Concarneau : on voit apparaître, par exemple, les signatures de P. Galabert jeune, ou de Cathala se mêler à l'ensemble des autres signatures.

L'objet de la requête du $1^{\text {er }}$ septembre 1767 , adressée " au Roi et à Nosseigneurs de son Conseil pour la pêche de sardine de Bretagne contre les particuliers marchands et négocians du Languedoc " est de mettre un point final à toutes ces tergiversations interminables de la part de l'État qui ne peuvent que favoriser la fraude. Les gens du Languedoc sont accusés, de même que les pêcheurs Basques du Labourd, de contrevenir " aux Arrêts du Conseil d'État de Sa Majesté des 24 août 1715, 7 octobre 1717, 18 novembre 1720 , et 24 août 1748 " en introduisant et permettant " l'entrée défendue des Sardines de Pêche étrangère dans le Royaume ${ }^{25}$ ". Le but des Bretons est de pousser l'État à prendre une décision ferme et définitive. Ils s'appuient sur les pièces précédentes du dossier (celles de 1749 et de 1754) qui attestent de l'ancienneté du contentieux pour, cette fois-ci, en finir une fois pour toute avec cette affaire :

"Ces pièces, en même temps qu'elles donneront connaissance de plusieurs faits concernant la Pêche de la sardine, éclairciront les prétentions et les raisons contentieuses et respectives des deux concurrences; et l'on ose espérer que la légitimité reconnue de l'une, fera la conviction de la bâtardise ou de l'illégitimité de l'autre ${ }^{26}$."

Il y a urgence pour les Bretons parce que la législation royale est partout ouvertement bafouée. Le 10 juillet 1767, l'intendant d'Auch, d'Etigny, un représentant de la monarchie donc, prend une ordonnance qui permet l'entrée à Saint-Jean-de-Luz et Ciboure, en Labourd, des sardines fraîches de pêche étrangère :

"C'est cette ordonnance qui a fondé l'introduction dans le Labourd des sardines étrangères. C'est elle qui l'a emporté sur toutes les observations mises sous les yeux du conseil ${ }^{27}$."

La hantise de voir le royaume inondé de sardines espagnoles, portugaises ou anglaises gagne du terrain en Bretagne :

"Un négociant de Bordeaux qui, des Sardines qu'il tire de Bretagne, fait chez lui un Entrepôt pour la consommation de plusieurs Villes du Languedoc, marque qu'en y faisant depuis peu sa tournée pour recueillir le payement de ses envois, la Sardine de Pêche étrangère, dans tous les endroits qu'il a parcourus, se trouvait chez tous les détaillans, s'y vendait publiquement et bouchait enfin le débit et la consommation de celle de Pêche de Bretagne qui désormais, par cette fraude ouverte, était dans le danger le plus imminent de succomber sous la masse énorme des Sardines étrangères, qui l'écraserait sans espérance de ressource si on n'y apportait un prompt et puissant remède ${ }^{28}$."

25. Arch. dép. d'Ille-et-Vilaine, C 3928, 1 septembre 1767.

26. Arch. dép. d'Ille-et-Vilaine, C 3928, $1^{\text {er }}$ septembre 1767.

27. Chambre de commerce de Bayonne, B.40, 5 septembre 1785.

28. Arch. dép. d'Ille-et-Vilaine, C 3928, $1^{\text {er }}$ septembre 1767. 
La fraude s'étend donc particulièrement au Pays basque, elle risque de faire tache d'huile dans toutes les régions du Midi et, par conséquent, de porter des coups encore plus rude à l'économie sardinière des côtes atlantiques de Bretagne :

" La Côte de Biscaye est à l'égard des lieux que l'on vient de citer, ce que celles du Comté de Nice, de Catalogne, Oran et Portugal, sont à l'égard de la Provence, du Languedoc et du Roussillon. A la Côte de Biscaye celle de Galice vient tout récemment ajouter un nouveau supplément d'abondance de Sardine dans votre Royaume ${ }^{29}$."

Les Bretons s'appuient alors, signe tangible de leur position de faiblesse, sur la législation protectionniste de la monarchie en matière de pêche : si l'État ne peut plus maîtriser les mécanismes d'une économie libérale et concurrentielle, il faut donc appliquer à la lettre les vieilles recettes du protectionnisme d'État et exécuter dans toute sa rigueur les dispositions de l'arrêt du 24 août 1748. Après une trop longue période de polémiques, les Bretons entendent mettre fin, en 1767, à cette affaire pénible en ramenant la monarchie à ses devoirs :

"Que la concurrence dont ces Particuliers [...] prétendent favoriser les Sardines de Pêche étrangère, n'étant fondée que sur le droit arbitraire qu'ils se sont arrogés eux-mêmes, n'est dans le fond que désobéissance et opposition à votre Autorité souveraine, au mépris de laquelle ils contreviennent impunément à l'Arrêt du Conseil de 1748. Que le terme étrangère énoncé dans l'Arrêt de 1748 devant s'étende à toute Pêche faite par autres que vos Sujets, il est clair que toute Sardine pêchée par les Espagnols, Portugais ou Anglais, sapoudrée ou salée, acquère dès lors la qualité d'étrangère, et se trouve conséquemment, suivant la précision du terme de l'Arrêt, dans le cas de la prohibition ${ }^{30}$."

Cela revient à dire qu'il faut taxer chaque contrevenant d'une amende de 3000 livres avec confiscation, qu'il soit Français ou d'autres nations. Cela revient à dire aussi que toutes sardines pêchées par autres que des Français, seront réputées sardines étrangères, c'est-à-dire fermées au marché national.

Quels ont été les résultats de ces plaintes? En 1767, le Midi continuait toujours de recevoir les sardines espagnoles et anglaises. En 1778, les Languedociens fondent leur demande sur le fait d'une disette de sardines en Bretagne en 1777, il en va de même en 1781 :

" La chambre de commerce de Montpellier sollicite la permission d'introduire pendant 6 mois seulement les sardines pressées et en saumure de pêche espagnole, sous prétexte que celle de Bretagne a absolument manqué ${ }^{31}$."

29. Arch. dép. d'Ille-et-Vilaine, C 3928.

30. Arch. dép. d'Ille-et-Vilaine, C 3928, $1^{\text {er }}$ septembre 1767.

31. Arch. dép. d'Ille-et-Vilaine, C 1596, 14 janvier 1781. 
Les Bretons, par l'intermédiaire de leur intendant, Caze de La Bove, sont encore sur la défensive lorsqu'ils déclarent au Contrôleur Général des Finances, Joly de Fleury, que tout cela n'est qu'un prétexte à la fraude :

" Il est vrai que la pêche n'a pas été très abbondante, mais on assure que les sardines qui ont été envoyées à Bordeaux restent invendues parce que les négociants de Narbonne, d'Agde et d'autres endroits du Languedoc ont espéré obtenir la permission d'en faire venir d'Espagne où ils se flattent de l'avoir à meilleur marché ${ }^{32}$."

En fait la fraude continue à s'installer partout. Les Anglais introduisent leurs sardines dans toute la Méditerranée, tant en France qu'en Espagne et en Italie. Non seulement il entre des sardines en fraude en Labourd, en Languedoc mais, ironie du sort, en Bretagne même, avec une sardine d'origine anglaise. Ce scandale éclate en Bretagne lorsque en 1773, le capitaine Martin Langeais amène à Sète de la sardine de fraude :

"Un bâtiment français, capitaine Martin Langeais, est arrivé au port de Cette avec un chargement de 650 grosses bariques de sardines pressées; les expéditions sont du bureau de Locmariaquer dépendant de celui de Vannes, et la déclaration porte que ces sardines viennent de Belle-Isle et des environs $^{33}$."

L'original du certificat des négociants fabricants de Belle-Île lave ces derniers de tous soupçons. La fraude est trop difficile dans les grands ports sardiniers bretons comme Belle-l̂le, Concarneau, Douarnenez ou Port-Louis qui sont tous surveillés :

" Les chargemens pour le Languedoc se font à Belle-Isle ou au Port-Louis ou dans cette rivierre. Ils se font en cueillette. C'est-à-dire que chaque fabricant ou négociant charge une certaine quantité de sardines soit pour son compte soit par une commission. Dès que le navire sera connu et qu'il sera en chargement il est donc très possible sans aporter d'entrave au commerce à former un État général par marque et par noms des chargeurs ${ }^{34}$."

La fraude ne peut se faire que dans des ports de second rang, là où les contrôles se font d'une manière moins stricte :

«Tous ceux [les chargements] qui ont été faits dans les autres petits ports de la côte, et principallement dans les rivierres de Vannes ou d'Auray, contenaient toujours en plus forte partie des sardines venant d'Espagne ou d'Angleterre, que l'on trouvait le secret de masquer, à la faveur d'une modique quantité de celles de Belle Isle ou du Port Louis, et à l'aide des certificats que l'on a toujours obtenus très facilement dans les petits ports de ces rivierres, pour constater ces chargements, qui, se faisant ordinairement à un certain éloignement de la terre, ne peuvent être vérifiés assez exactement par les officiers publics qui délivrent ces certificats, et qui sont obligés, par cette raison, de s'en rapporter à la déclaration où des chargeurs ou des capitaines des navires et batiments qui les trompent. Il est certain que c'est ainsi que l'année dernière on parvint à masquer dans la rivierre de

32. Arch. dép. d'Ille-et-Vilaine, C 1596, 25 janvier 1782.

33. Arch. dép. d'Ille-et-Vilaine, C 1595, 2 octobre 1773.

34. Arch. dép. d'Ille-et-Vilaine, C 1595, 16 novembre 1773. 
Vannes un parti considérable de sardines de pêche anglaise, qui fut introduit à Cette, pour des sardines de Belle Isle et du Port Louis, et cette fraude a fait un tort considérable à la pêche de Bretagne, qui est d'ailleurs devenue très ingrate par la cherté des rogues ${ }^{35}$."

Ce sont donc des ports, principalement ceux du Golfe du Morbihan, que la fraude se fait. Ce n'est pas, évidemment, une affaire de fabricants bretons de sardines pressées d'autant plus que ces ports du Golfe du Morbihan n'ont jamais pratiqué la pêche de la sardine, pas plus qu'ils n'ont opéré dans sa transformation; en revanche ce sont eux qui fournissent aux ports sardiniers bretons une grande partie de la flottille de chasse-marée qui acheminent les sardines vers Bordeaux ou le Midi. Ces ports ont donc une tradition de commerce et pour alimenter leur négoce ils ne craignent pas de fermer les yeux sur des actions délictueuses. La méthode de la fraude est simple, il suffit de couvrir la marchandise anglaise de quelques barriques de pêche bretonne :

"Un navire qui avait, dit-on, chargé dans ses parages, arrive à Cette. Il avait sur son chargement quelques barils de sardinnes de pêche française et en dessous 600 barriques de Bordeaux de sardines anglaises [...] nos fabricans ne sont point dans l'usage de mettre leurs sardinnes en bariques de Bordeaux ${ }^{36}$."

Le manque de décision de l'État a incontestablement entretenu ce climat de fraude mais cela n'explique pas tout. Dans un monde de pénurie, ces événements montrent finalement qu'une loi, même imposée de manière autoritaire par les représentants de l'État, ce qui n'a jamais été le cas ici, ne peut empêcher à la fraude de se manifester : les besoins de la population ont été trop forts, les espaces maritimes trop larges et le désir de fraude, pour de bonnes ou mauvaises raisons, trop universellement présent.

35. Arch. dép. d'Ille-et-Vilaine, C 1595, 20 novembre 1773. 36. Arch. dép. d'Ille-et-Vilaine, C 1595, 16 novembre 1773. 


\section{RÉSUMÉ}

On trouve aux Archives départementales d'Ille-et-Vilaine un dossier datant de 1767 qui fait état d'une longue polémique entre Bretons et gens du Languedoc au sujet de la sardine pressée : les Méditerranéens sont accusés par les Bretons de leur faire une concurrence déloyale en important des sardines de pêche étrangère. Les Bretons s'appuient en cela sur l'arrêt du Conseil royal du 24 août 1748 qui leur donne le monopole de la commercialisation de la sardine dans tout le royaume. Les gens du Languedoc, très dépendants de cette ressource, préféreraient importer la sardine de la Catalogne voisine voire des États italiens, qui correspond mieux à leurs goûts et qui est moins chère.

Il est évident que face à une monarchie souvent hésitante sur la conduite à tenir, la fraude ne cesse de s'étendre. De fait, les Bretons ont fort à faire pour défendre leurs droits légitimes.

\section{ABSTRACT}

A file dating back to 1767 can be found in the "Archives départementales d'Ille et Vilaine". It reports a long polemic between Bretons and Languedoc inhabitants about pressed sardines. Bretons accused the Southerners of competing unfairly with them by importing sardines fished abroad. To do so, they relied on the ruling of the royal council declared on $24^{\text {th }}$ August 1748 which gave them the monopoly in trading sardines all over the kingdom. Languedoc inhabitants who were extremely dependent on this resource, wished they could import sardines from neighbouring Catalonia, or even from Italy, since they were cheaper and suited them better.

However, as the monarchy often wavered about how to deal with that, smuggling kept increasing. Thus, Bretons had a hard job trying to defend their legitimate rights. 